\title{
LOW-POWER SOLAR FLARES OF OPTICAL AND X-RAY WAVELENGTHS FOR SOLAR CYCLES 21-24
}

\author{
A.V. Borovik \\ Institute of Solar-Terrestrial Physics SB RAS, \\ Irkutsk,Russia,aborovik@iszf.irk.ru
}

\author{
A.A. Zhdanov \\ Institute of Solar-Terrestrial Physics SB RAS, \\ Irkutsk, Russia, kick.out@mail.ru
}

\begin{abstract}
Using data obtained in optical and X-ray wavelengths, we have analyzed solar flare activity for cycles 21-24. Over the last three cycles, solar activity is shown to decrease significantly. As compared to solar cycle 21 (the most active over the last 50 years), in cycle 24 2-4-class large optical flares are 4.4 times rarer; 1-class flares, 8.2 times; and S-class small flares, 4.1 times. The number of X-class flares decreased 3.7 times; M-class flares, 3.2 times. This confirms that secular solar activity trends affect peak values of 11year cycles. It is shown that optical low-power flares can be accompanied by proton fluxes and X-ray bursts of different intensity, including X-class ones. Ranges of small flare emission in soft X-rays largely overlap with emission ranges of flares of high optical classes. We
\end{abstract}

have confirmed that X-ray emission from solar flares appears on average $2 \mathrm{~min}$ before the optical emission. The X-ray maximum for small optical flares and 1-class flares occurs approximately $1 \mathrm{~min}$ later; for 2-4-class flares, $2 \mathrm{~min}$.

Keywords: solar activity, solar flares.

\section{INTRODUCTION}

Solar flares are accompanied by electromagnetic emission in a wide range of wavelengths - from solar radio emission to gamma-ray emission - by accelerated particle fluxes, coronal mass ejections. Propagating in interplanetary space, the flow of ionized matter generates a powerful shock wave, disturbs the geomagnetic field, causes magnetic storms, has an effect on the state of Earth's atmosphere.

There are two main classifications of solar flares that complement each other: in optical and X-ray wavelength ranges.

As derived from optical observations in the $\mathrm{H} \alpha$ line, according to the International classification [Smith, Smith, 1966; Altyntsev et al., 1982; Temmer et al., 2001], flares, in terms of area, fall into five classes $(\mathrm{S}, 1,2,3,4)$; in terms of emission intensity, into three: faint $(\mathrm{F})$, normal $(\mathrm{N})$, and bright (B). Combination of these parameters (area and intensity) makes up the optical importance of flare (Table 1).

The flare area is expressed in square degrees (sq. deg), millionths of solar disk (MD) or millionths of solar hemisphere $(\mathrm{MH})$. In the disk center, 1 sq. $\operatorname{deg}=97 \mathrm{MD}$ $=48.5 \mathrm{MH}$. The flare intensity is usually defined in terms of the units of the adjacent undisturbed chromosphere

Table 1

Classification of solar flares by area and emission intensity in the $\mathrm{H} \alpha$ line

\begin{tabular}{|r|r|r|r|r|}
\hline \multicolumn{2}{|c|}{ Area } & \multicolumn{3}{c|}{ Relative intensity } \\
\hline sq. deg & $\mathrm{MH}$ & $\mathrm{F}$ & $\mathrm{N}$ & $\mathrm{B}$ \\
\hline$\leq 2.0$ & $\leq 100$ & $\mathrm{SF}$ & $\mathrm{SN}$ & $\mathrm{SB}$ \\
\hline $2.1-5.1$ & $100-250$ & $1 \mathrm{~F}$ & $1 \mathrm{~N}$ & $1 \mathrm{~B}$ \\
\hline $5.2-12.4$ & $250-600$ & $2 \mathrm{~F}$ & $2 \mathrm{~N}$ & $2 \mathrm{~B}$ \\
\hline $12.5-24.7$ & $600-1200$ & $3 \mathrm{~F}$ & $3 \mathrm{~N}$ & $3 \mathrm{~B}$ \\
\hline$>24.7$ & $>1200$ & $4 \mathrm{~F}$ & $4 \mathrm{~N}$ & $4 \mathrm{~B}$ \\
\hline
\end{tabular}

intensity. A flare with a relative intensity $160-260 \%$ is considered faint $(\mathrm{F}) ; 260-360 \%$, normal $(\mathrm{N})$; over $360 \%$, bright (B).

In the X-ray band, the flare class is set depending on the maximum X-ray burst amplitude in the energy range $0.5-10 \mathrm{keV}$ according to GOES measurements in the range $1-8 \AA$ (Table 2 ).

Table 2

Classification of flares by X-ray burst amplitude (GOES classification)

\begin{tabular}{|c|c|c|c|c|c|}
\hline Flare class & A & B & C & M & X \\
\hline $\begin{array}{c}\text { X-ray } \\
\text { burst } \\
\text { amplitude, } \\
\text { W/m }\end{array}$ & trom $10^{-8}$ & from $10^{-7}$ & from $10^{-6}$ & from $10^{-5}$ & from $10^{-4}$ \\
to $9.9 \cdot 10^{-7}$ & to $9.9 \cdot 10^{-6}$ & to $9.9 \cdot 10^{-5}$ & to $30 \cdot 10^{-4}$ \\
A1-A9 & B1-B9 & C1-C9 & M1-M9 & X1-X30 \\
\hline
\end{tabular}

X-ray classes A-M are divided into nine subclasses (from 1 to 9). Each next class represents a 10-fold increase in importance of a flare with respect to the previous one, i.e. an M1 flare is 10 times more powerful than $\mathrm{C} 1$; and an $\mathrm{X} 1$ flare is 10 times stronger than M1. Since the amplitude of the X-ray burst in the strongest X-ray flare ever recorded was $28 \times 10^{-4} \mathrm{~W} / \mathrm{m}^{2}$ (the November 04, 2003 flare), the $\mathrm{X}$ class was divided into 30 subclasses (from $10^{-4}$ to $30 \cdot 10^{-4} \mathrm{~W} / \mathrm{m}^{2}$ ).

\section{FEATURES OF FLARE ACTIVITY IN SOLAR CYCLES 21-24}

According to the International Flare Patrol data [http://www.ngdc.noaa.gov/stp/space-weather/solardata/solar-features/solar-flares/h-alpha/events/], 122750 optical flares occurred on the Sun in cycles 21-24, 109687 of which were small flares $(\mathrm{SmF})$ of $\mathrm{S}$ class, 
11281 were 1-class flares, and 1782 were large flares of 2-4 classes (Table 3). SmF comprised $90 \%$ of all flares; large flares, $1.5 \%$.

Table 3

Number of optical and X-ray solar flares that occurred in cycles 21-24

\begin{tabular}{|c|c|c|c|c|c|c|c|c|}
\hline No. of & Period & \multicolumn{4}{|c|}{ X-ray class } & \multicolumn{3}{|c|}{ Optical class } \\
\cline { 3 - 9 } cycle & & $\mathrm{B}$ & $\mathrm{C}$ & $\mathrm{M}$ & $\mathrm{X}$ & $\mathrm{S}$ & 1 & $2-4$ \\
\hline 21 & $\begin{array}{c}06.1976- \\
09.1986\end{array}$ & 2856 & 14561 & 2174 & 165 & 46694 & 5514 & 648 \\
\hline 22 & $\begin{array}{c}09.1986- \\
05.1996\end{array}$ & 6086 & 12433 & 2020 & 152 & 31695 & 3430 & 584 \\
\hline 23 & $\begin{array}{c}05.1996- \\
01.2009\end{array}$ & 7974 & 13073 & 1437 & 122 & 19974 & 1662 & 404 \\
\hline 24 & $\begin{array}{c}01.2009- \\
06.2017\end{array}$ & 5388 & 7505 & 688 & 45 & 11324 & 675 & 146 \\
\hline $21-24$ & $\begin{array}{c}06.1976- \\
06.2017\end{array}$ & 22304 & 47572 & 6319 & 484 & 109687 & 11281 & 1782 \\
\hline
\end{tabular}

As derived from GOES data [https://www.ngdc. noaa.gov/stp/space-weather/solar-data/solar-features/solar-flares/X-rays/goes/xrs/], 76679 flares occurred during the four cycles; 22304 of them are B-class flares, 47572 are C-class flares, 6319 are M-class flares, and 484 are X-class flares.

Note that according to the method of detecting X-ray flares with GOES satellites [https://www.ngdc.noaa. gov/stp/space-weather/solar-da-ta/solar-features/solarflares/documentation/readme_so-lar-features_solarflares.pdf], the event is considered real if during four successive 1-min intervals three conditions hold [Veronig et al., 2002]:

- the X-ray flux is constantly increasing;

- the X-ray flux is higher than B1;

- the last value of the X-ray flux is 1.4 times or more larger than that recorded three minutes earlier.

Probably for this reason, in GOES catalogs A-class flares comprise only $\sim 0.014 \%$ of the total number of flares.

The X-ray class of a flare is determined from the flux value at the point of maximum. The maximum $\mathrm{X}$ ray flux is found by 1-min averaging.

Since the level of the background total solar emission during a flare can change and other events can occur during the decay phase, it is rather difficult to determine the time of X-ray flux recovery to the pre-flare level. Therefore, the time of the X-ray flare end is defined as the time of return of the flux to half of its peak value.

During the four cycles, solar flare activity gradually decreased (Table 3, Figures 1-3). As compared to solar cycle 21 (the most active over the last 50 years), in cycle 24 in the optical range small flares were recorded 4.1 times rarer; 1-class flares, 8.2 times; and 2-4-class flares, 4.4 times. The number of X-class flares decreased 3.7 times; M-class, 3.2 times; C-class, 1.9 times.

The decrease in sunspot activity over the last four 11-year cycles occurred during the decay phase of the 100 -year solar cycle and was probably linked to global trends in solar activity.

The background solar emission significantly affects the recording of X-ray flares, especially B-class ones. Observations show that during solar minimum the background level is within $\mathrm{A}<1$; during solar maximum,

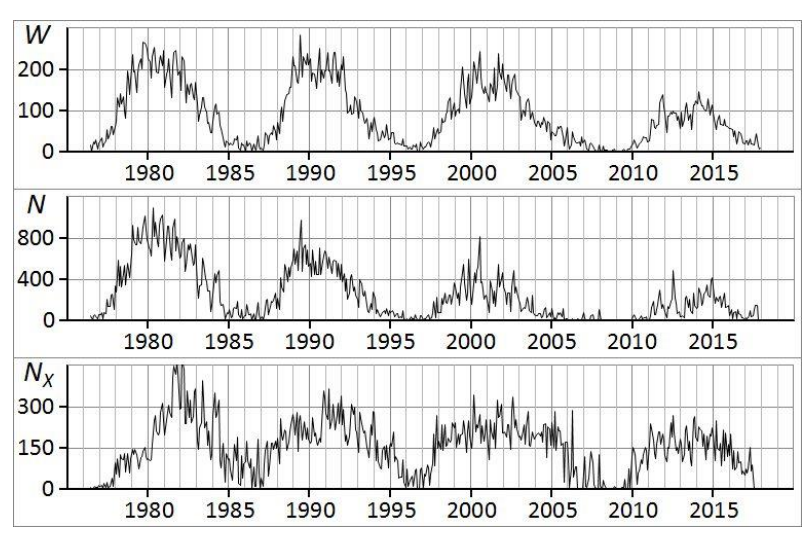

Figure 1. Distribution of the number of optical $(N)$ and Xray $\left(N_{\mathrm{X}}\right)$ flares over years in solar cycles $21-24 . W$ is Wolf numbers

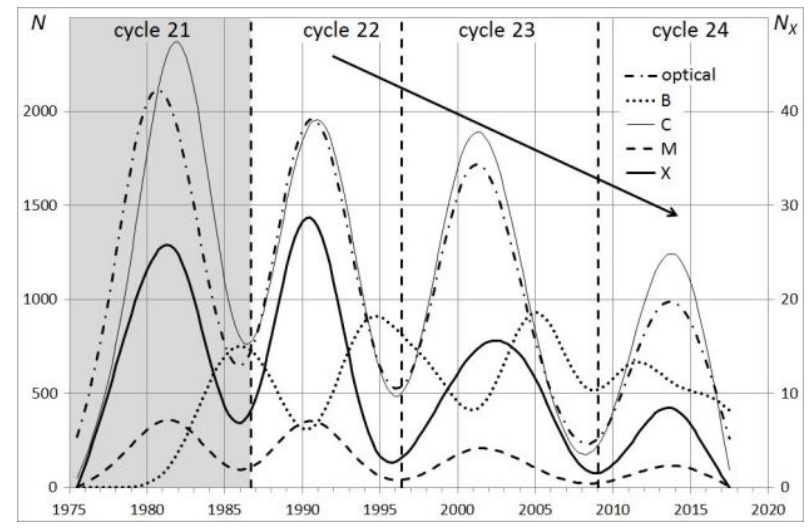

Figure 2. Distribution of the number of optical $(N)$ and Xray $\left(N_{\mathrm{X}}\right)$ flares of different classes in cycles $21-24$ over years (smoothed curves)

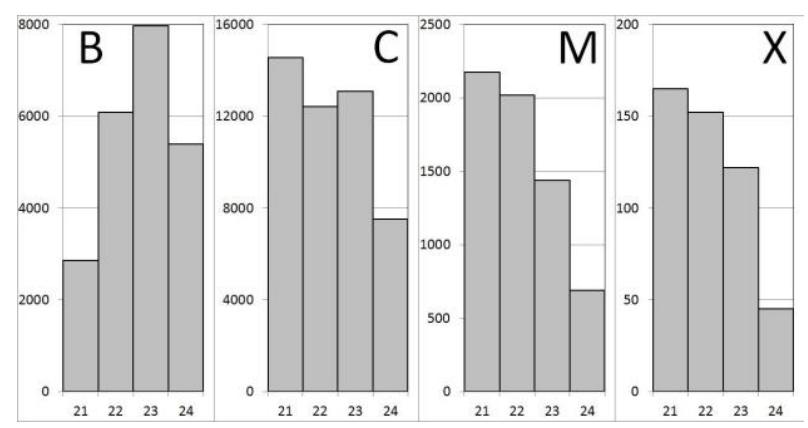

Figure 3. Distribution of the number of X-ray flares of different classes over cycles

it increases to $\mathrm{C}<5$; and during specific periods it can reach the M-level (Figure 4).

During cycles 21-24 due to cyclic variations of the Xray background level, the number of recorded B-class Xray flares varied almost in antiphase with flares of higher $\mathrm{X}$-ray classes - it decreased at solar maximum and increased at solar minimum (Figure 2).

This fact is also noted in [Sotnikova, Moskalenko, 1999]. However, the number of B flares increased with each cycle (Figure 3), thus probably indicating a gradual attenuation of the background solar emission. Table 4 shows the number of flares of respective X-ray and optical classes for cycles 21-24 and provides information on solar proton events [https://umbra.nascom.nasa.gov/SEP] over the period from 1976 to 2017. 


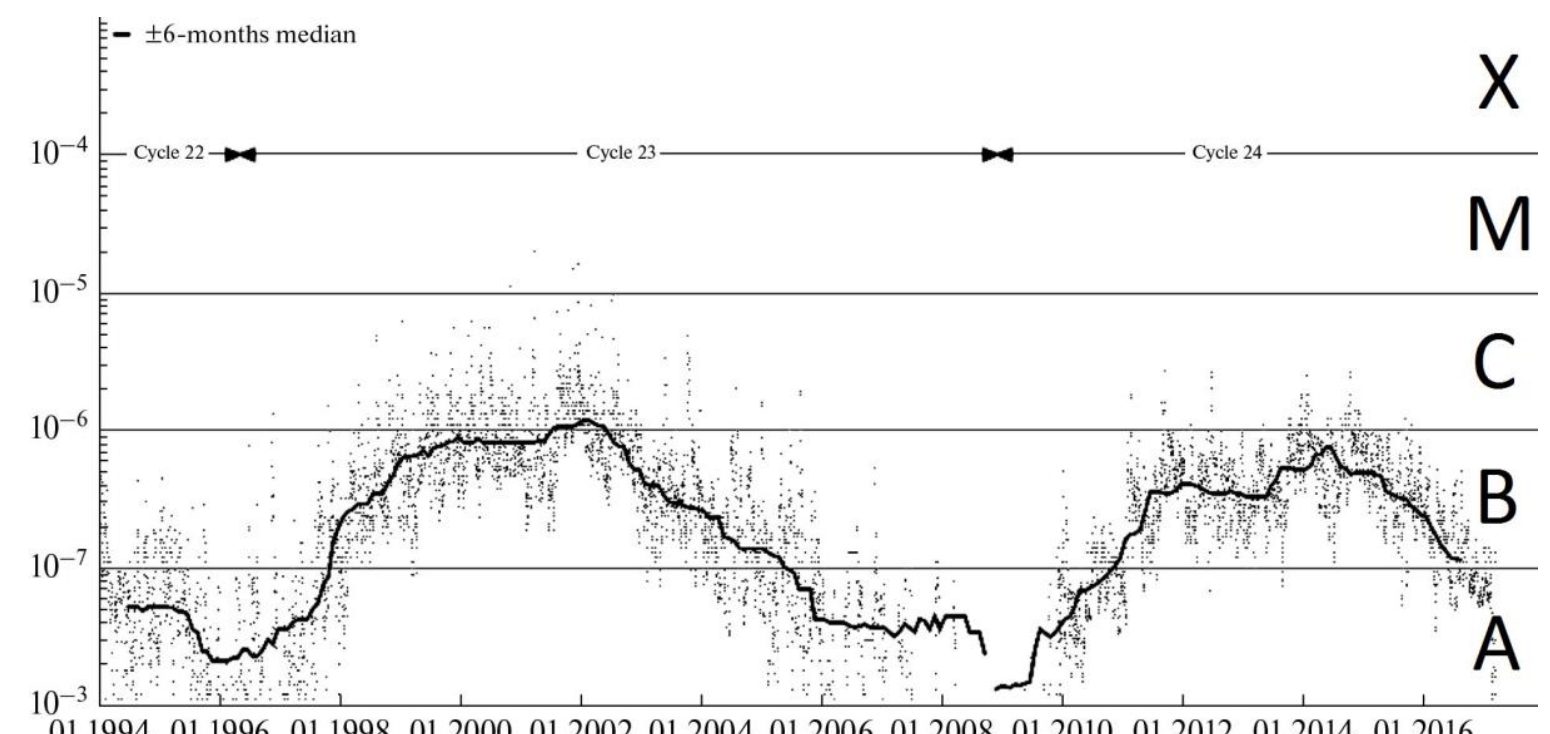

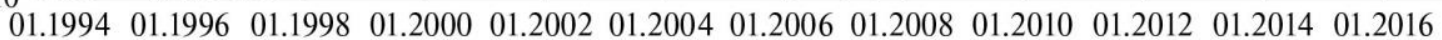

Figure 4. Variations in the background solar emission in cycles 23-24 [https://sidstation.loudet.org/solar-activity-en.xhtml]

Table 4

X-ray flares and proton events in solar cycles $21-24$

\begin{tabular}{|c|c|c|c|c|c|c|c|c|}
\hline \multirow[t]{2}{*}{$\mathrm{H} \alpha$} & \multicolumn{4}{|c|}{ X-ray flares } & \multicolumn{4}{|c|}{$\begin{array}{l}\text { Proton events } \\
E>10 \mathrm{MeV}\end{array}$} \\
\hline & B & $\mathrm{C}$ & M & $\mathrm{X}$ & B & C & M & $\mathrm{X}$ \\
\hline SF & 3231 & 13318 & 720 & 16 & & 2 & 3 & \\
\hline SN & 326 & 4309 & 477 & 6 & & 1 & 2 & \\
\hline SB & 36 & 1479 & 447 & 10 & & & 2 & 1 \\
\hline $1 \mathrm{~F}$ & 25 & 947 & 268 & 8 & & 3 & 6 & 3 \\
\hline $1 \mathrm{~N}$ & 7 & 1217 & 764 & 17 & & & 12 & 5 \\
\hline 1B & 8 & 548 & 914 & 57 & & & 5 & 6 \\
\hline $2 \mathrm{~F}$ & 1 & 38 & 49 & 1 & & 2 & & \\
\hline $2 \mathrm{~N}$ & & 75 & 191 & 16 & & 1 & 14 & 9 \\
\hline $2 \mathrm{~B}$ & & 43 & 357 & 159 & & & 21 & 30 \\
\hline $3 \mathrm{~F}$ & & & & & & & & \\
\hline $3 \mathrm{~N}$ & & 2 & 6 & 6 & & & 2 & \\
\hline $3 \mathrm{~B}$ & & 2 & 26 & 67 & & & 9 & 33 \\
\hline $4 \mathrm{~F}$ & & & & & & & & \\
\hline $4 \mathrm{~N}$ & & & & & & & & \\
\hline $4 \mathrm{~B}$ & & & 3 & 6 & & & 2 & 5 \\
\hline In tota & & & & & & & & \\
\hline $\mathrm{S}$ & 3593 & 19106 & 1644 & 32 & & 3 & 7 & 1 \\
\hline 1 & 40 & 2712 & 1946 & 82 & & 3 & 23 & 14 \\
\hline $2-4$ & 1 & 160 & 632 & 255 & & 3 & 48 & 77 \\
\hline
\end{tabular}

The data indicates (Table 4) that all optical flares, including $\mathrm{SmF}$, can be accompanied by proton fluxes and $\mathrm{X}$ ray bursts of different intensity. Of 179 recorded proton events, $6 \%$ (11) were optical SmF of S class, $22 \%$ (40) were 1-class flares, and $72 \%$ (128) were large flares of 24 classes. Of 369 X-class flares, $9 \%$ were small optical flares (S), $22 \%$ were 1-class flares, $69 \%$ were large flares (2-4); whereas among 4222 M-class flares, 39, 46, and 15 $\%$ respectively.

With increasing optical class of flares, the intensity of an accompanying X-ray burst tends to increase. Moreover, the power distributions in the X-ray flux of S-, 1-, and 2-4class flares largely overlap, and their scope covers the vast majority of X-ray emission levels (Figure 5).

\section{RELATIONSHIP BETWEEN OPTICAL AND X-RAY SOLAR FLARES}

Solar flares have been studied by many authors on the basis of satellite measurements in different wavelengths of soft X-rays. A similarity has been found between time profiles of X-ray and optical emission of flares; mean time parameters of emission have been determined; for flares of different optical classes, rough (typical) values of energies and amplitude of respective X-ray bursts have been identified, etc. [Culhane, Phillips, 1970; Drake, 1971; Thomas, Teske, 1971; Pearce, Harrison, 1988; Kurt, 1990; Veronig et al., 2002; Fletcher et al., 2011]. However, when evaluating, e.g., the correspondence between optical and X-ray flares, statistics did not exceed several hundreds of flares. Taking into account that there were no additional studies in this area in recent decades, we have set a goal to try to more reliably correlate optical solar flares to X-ray ones, using extensive observational data from GOES satellites.

Data Selection. Analysis of GOES satellite data shows that it is often heterogeneous and incomplete. As follows from different sources, solar X-ray emission has been measured by GOES satellites more or less regularly since 1975. Until 1986, X-rays were recorded by at least one GOES satellite. There were also technical problems that were overcome with the launch of the GOES- 8 satellite in 1994 [Veronig et al., 2002].

Until 1997, catalogs of X-ray flares contained information on time parameters of optical flares and no data on the intensity of the total radiant flux. Over the period from January 2004 to March 2007, there was no data on importance of optical flares. Also doubtful is homogeneity and completeness of the data from the beginning to approximately the middle of solar cycle 21. In this period, a relative number of X-ray flares of B and $\mathrm{C}$ classes show an atypical behavior (Figure 6).

Probably during the first flights of GOES satellites, measurements were experimental for a time, and this affected homogeneity of the data and the total statistics 

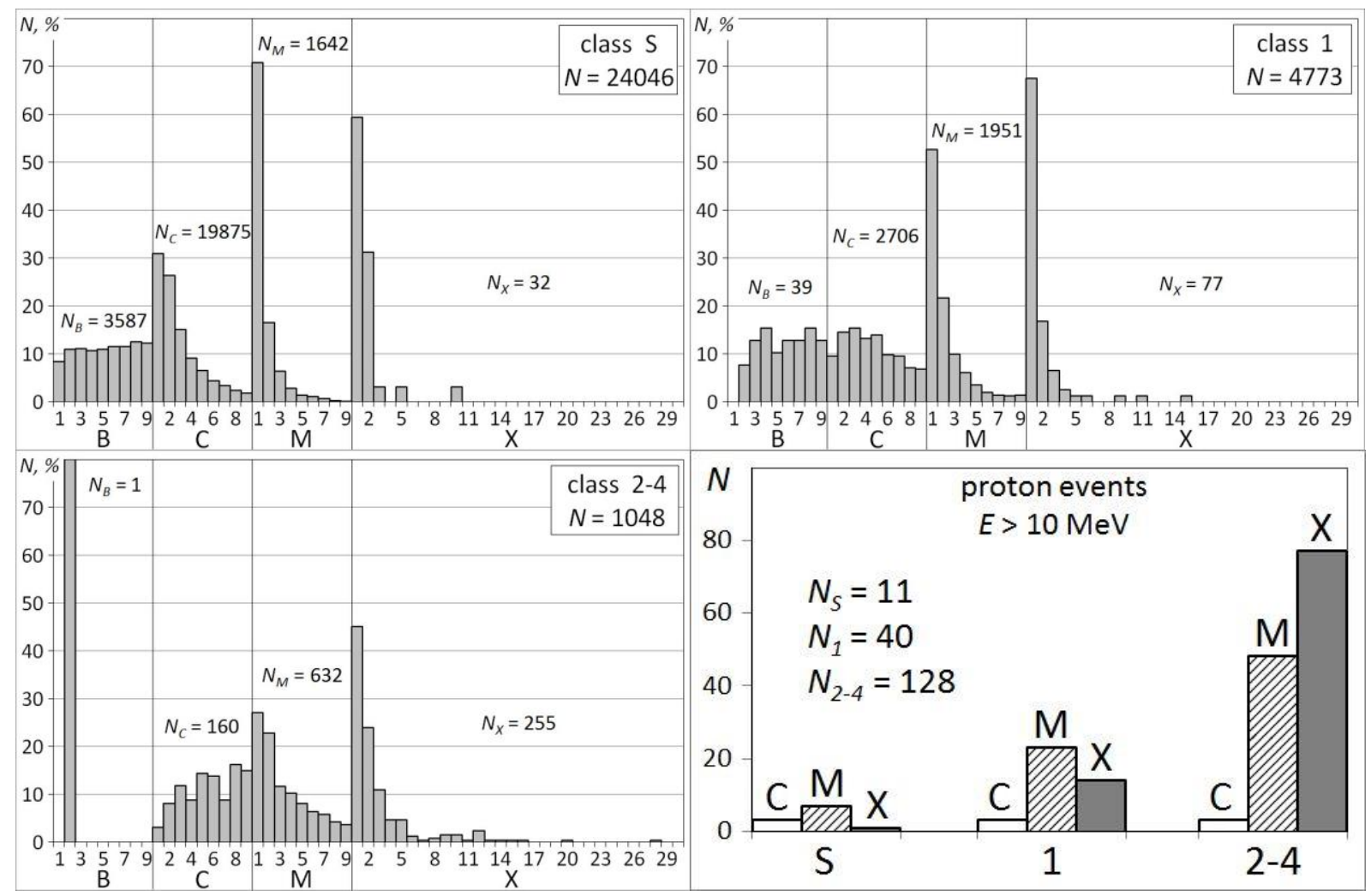

Figure 5. Distribution of the number of events in S-, 1-, and 2-4-class optical flares in solar cycles 21-24 cycles (GOES data): two top panels and the right bottom panel are X-ray events (in \%), the distributions are built separately for each X-ray class; the left bottom panel indicates proton events

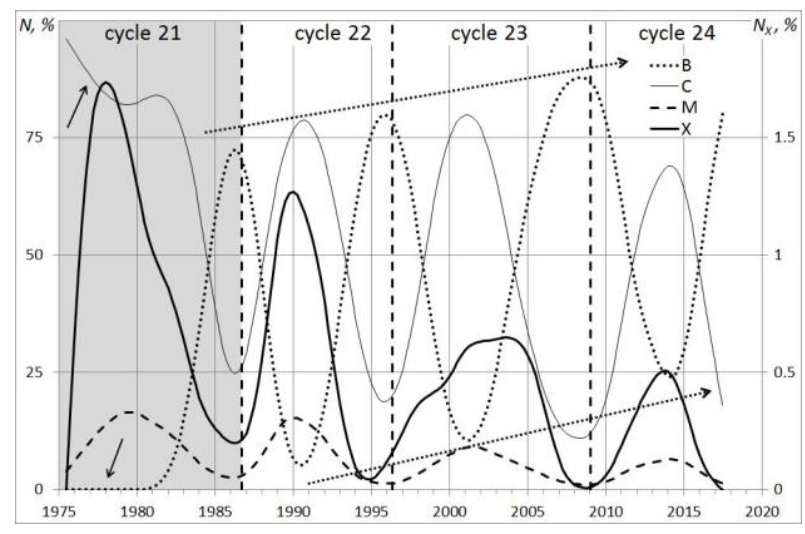

Figure 6. Relative distribution of the number of X-ray flares in solar cycles 21-24 (smoothed curves). The right axis is the relative number of X-class flares $\left(N_{\mathrm{X}}, \%\right)$

of X-ray flares. Therefore, to solve this problem we have restricted the statistical series to the data obtained during solar cycles 22-24 (September 1986-June 2017).For this time interval, we have performed a correlation analysis of the number of optical and X-ray flares of each class over months. The results showed a high correlation between optical $\mathrm{SmF}$, 1-class flares and $\mathrm{C}$ and M-class X-ray flares. Large optical flares of 2-4 classes have high correlation coefficients with $\mathrm{M}$ - and $\mathrm{X}$-class X-ray flares (Table 5).

Figure 7 presents correlation coefficients between S-, 1-, 2-4-class optical flares and their associated peak values of X-ray bursts. Histograms show a substantial overlap in intensity of X-ray bursts accompanying optical flares. Here, we can see maxima in C class (shifted with increasing optical class of flare toward an increase in X-ray emission intensity), $\mathrm{M}$ class (practically identical for flares of all optical classes), and $\mathrm{X}$ class (for large optical flares).

\section{TIME CHARACTERISTICS OF X-RAY AND OPTICAL EMISSION OF FLARES}

In order to establish to which solar event the X-ray burst belongs, the time of onset of an X-ray burst is compared with that of an optical flare from data obtained in the $\mathrm{H} \alpha$ line.

As noted above, time parameters of X-ray bursts have become available in the GOES database since 1997. To independently compare time characteristics of optical and Xray flares, we have developed a technique that allows software to compare X-ray and optical events. To do this, from the GOES database we excluded the flares which had no exact start time of X-ray burst. Because of possible errors in identifying the optical importance of flares near the limb, we also omitted the flares whose longitude relative to the central meridian exceeded $65^{\circ}$.

Then, from the database [http://www.ngdc.noaa.gov/ stp/space-weather/solar-data/solar-features/solar-flares/ halpha/events/], we selected optical flares occurring in the interval of $\pm 10 \mathrm{~min}$ from the onset of the X-ray burst from GOES data. If in this interval there was more than one optical flare, such an X-ray flare was ignored. If there was 
Table 5

Correlation coefficients between optical and X-ray classes of solar flares

\begin{tabular}{|c|c|c|c|c|c|}
\hline Cycle number & Period & $\mathrm{S} \leftrightarrow \mathrm{B}$ & $\mathrm{S} \leftrightarrow \mathrm{C}$ & $\mathrm{S} \leftrightarrow \mathrm{M}$ & $\mathrm{S} \leftrightarrow \mathrm{X}$ \\
\hline 22 & $09.1986-05.1996$ & -0.60 & 0.88 & 0.78 & 0.56 \\
\hline 23 & $05.1996-01.2009$ & -0.31 & 0.87 & 0.77 & 0.32 \\
\hline 24 & $01.2009-06.2017$ & -0.30 & 0.81 & 0.68 & 0.43 \\
\hline $22-24$ & $09.1986-06.2017$ & -0.39 & 0.83 & 0.77 & 0.48 \\
\hline Cycle number & Period & $1 \leftrightarrow \mathrm{B}$ & $1 \leftrightarrow \mathrm{C}$ & $1 \leftrightarrow \mathrm{M}$ & $1 \leftrightarrow \mathrm{X}$ \\
\hline 22 & $09.1986-05.1996$ & -0.62 & 0.78 & 0.88 & 0.71 \\
\hline 23 & $05.1996-01.2009$ & -0.30 & 0.82 & 0.87 & 0.45 \\
\hline 24 & $01.2009-06.2017$ & -0.38 & 0.81 & 0.84 & 0.56 \\
\hline $22-24$ & $09.1986-06.2017$ & -0.40 & 0.72 & 0.86 & 0.62 \\
\hline Cycle number & Period & $2-4 \leftrightarrow \mathrm{B}$ & $2-4 \leftrightarrow \mathrm{C}$ & $2-4 \leftrightarrow \mathrm{M}$ & $2-4 \leftrightarrow \mathrm{X}$ \\
\hline 22 & $09.1986-05.1996$ & -0.58 & 0.72 & 0.90 & 0.83 \\
\hline 23 & $05.1996-01.2009$ & -0.25 & 0.63 & 0.81 & 0.62 \\
\hline 24 & $01.2009-06.2017$ & -0.35 & 0.68 & 0.90 & 0.69 \\
\hline $22-24$ & $09.1986-06.2017$ & -0.39 & 0.66 & 0.89 & 0.76 \\
\hline
\end{tabular}

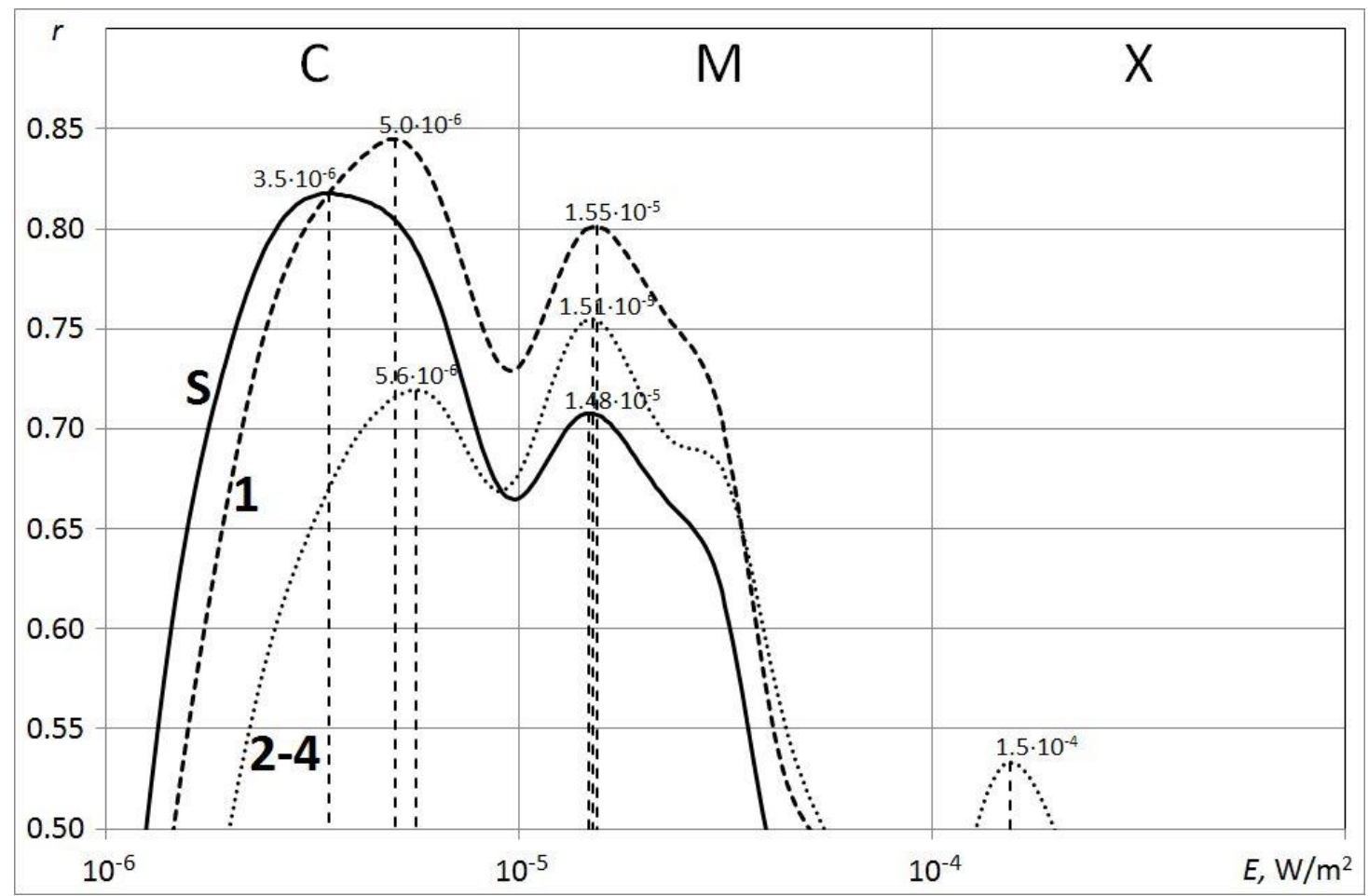

Figure 7. Correlation coefficients between the class of optical flares and the maximum amplitude of an X-ray burst (by months, September 1986 - June 2017)

only one optical flare, its coordinates and importance were compared with those given in the GOES database. If its optical importance did not match and the difference between the coordinates exceeded $5^{\circ}$, the X-ray flare was excluded from consideration. If all checks were successful, the X-ray burst was thought to be generated by this optical flare. The selection results indicate that $\sim 25 \%$ of X-ray flares in the GOES catalogs are not confidently identified with optical flares.

From the created database, we determined the difference in time between flare onsets (maxima) in the optical and X-ray emission (Figure 8). Time parameters in the catalogs of optical and X-ray flares are accurate to the minute.

According to the results, X-ray emission occurs $\sim 2 \mathrm{~min}$ before the optical emission regardless of the optical im- portance of flare, which is consistent with the results obtained in [Thomas, Teske, 1971]. The maximum emission in the X-ray range for SmF and 1-class flares occurs approximately by $1 \mathrm{~min}$ later than that in the optical range; for 2-4-class flares, by 2 min later.

\section{CONCLUSIONS}

In this paper, we have analyzed flare activity during solar cycles 21-24. We have shown that over the period the number of solar flares recorded in optical and X-ray ranges gradually decreased, which might have been due to the influence of the global trend in solar activity (secular solar cycle) on the 11-year solar cycles. However, GOES data has revealed that the number of B-class X-ray flares increased from cycle to cycle, thus suggesting that the solar $\mathrm{X}$-ray background gradually decreased. We have found 

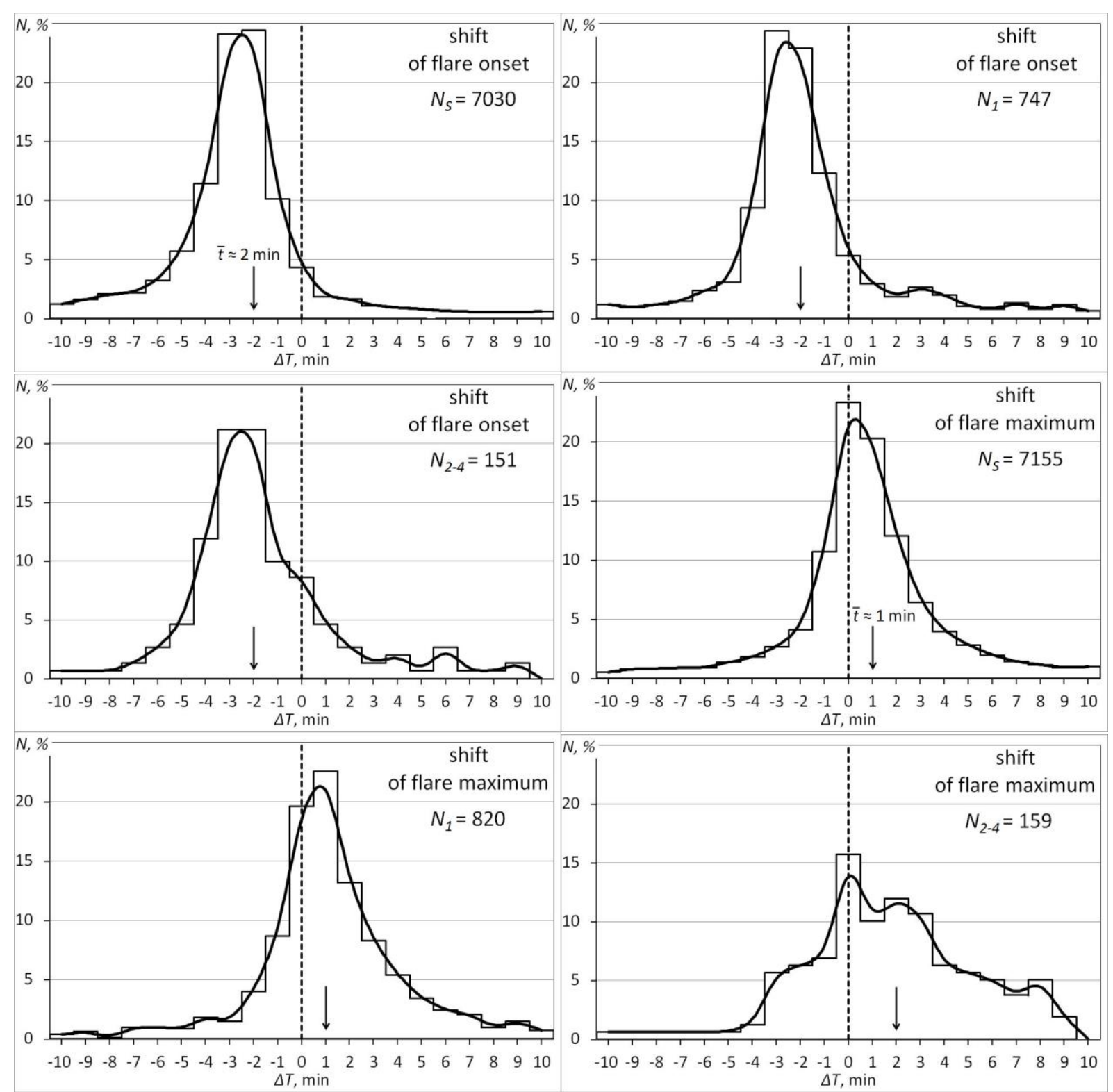

Figure 8. Distributions of time intervals between onsets (maxima) of a flare in optical and X-ray ranges. The dashed vertical line indicates the onset of an optical flare; the arrow, mean histogram

that low-power optical S-class solar flares are accompanied by proton fluxes and X-ray emission of different intensity, including X-class one. For optical flares of different classes, soft X-ray ranges have been shown to overlap to a great extent. We have developed a technique capable of correlating optical and X-ray flares, which has demonstrated a high degree of reliability. From the database created using this technique, we have determined mean times of onset and maximum emission of optical and X-ray flares. We have found that regardless of optical class of flare the $\mathrm{X}$-ray emission appears on average $2 \mathrm{~min}$ before the optical one. The maximum X-ray emission for small optical flares of $S$ class and 1-class flares occurs about 1 min later than the maximum optical emission; for 2-4-class flares, 2 min later.

The work was performed with budgetary funding of Basic Research program II.16 and was supported by RFBR grant No. 19-52-45002.

\section{REFERENCES}

Altyntsev A.T., Banin V.G., Kuklin G.V., Tomozov V.M. Solnechnye vspyshki [Solar Flares]. Moscow, Nauka Publ., 1982, 246 p. (In Russian).

Culhane J.L., Phillips K.J.H. Solar X-ray bursts at energies less than $10 \mathrm{keV}$ observed with OSO-4. Solar Phys. 1970, vol. 11, iss. 1, pp. 117-144. DOI: 10.1007/BF00156556.

Drake J.F. Characteristics of soft solar X-ray bursts. Solar Phys. 1971, vol. 16, iss. 1, pp. 152-185. DOI: 10.1007/ BF00154510.

Fletcher L., Dennis B.R., Hudson H.S., Krucker S., Phillips K., Veronig A., Battaglia M., et al. An observational overview of solar flares. Space Sci. Rev. 2011, vol. 159, iss. 14, pp. 19-106. DOI: 10.1007/s11214-010-9701-8.

Kurt V.G. Electrons and X-ray emission of solar flares. Basic Plasma Processes on the Sun: Proc. 142th Symposium of the International Astronomical Union. 1990, pp. 409-413. DOI: 10.1017/S007418090008832X.

Pearce G.A., Harrison R.A. Statistical analysis of the soft X-ray profiles of solar flares. Astron. Astrophys. 1988, vol. 
206, no. 1, pp. 121-128.

Smith G., Smith E. Solnechnye vspyshki [Solar Flares]. Moscow, Mir Publ., 1966, 426 p. (In Russian). English edition: Smith H.J., Smith E.P. Solar Flares. New York, Macmillan; London, Collier-Macmillan, 1963, 322 p.

Sotnikova R.T., Moskalenko A.V. The Sun in X-rays. Trudy VII simpoziuma po solnechno-zemnoi fizike Rossii $i$ stran SNG [Proc. VIIth Symposium on Solar-Terrestrial Physics for Russia and CIS Countries]. Troitsk, 1999, pp. 156-161. (In Russian).

Temmer M., Veronig A., Hanslmeier A., Otruba W., Messerotti M. Statistical analysis of solar $\mathrm{H} \alpha$ flares. Astron. Astrophys. 2001, vol. 375, no. 3, pp. 1049-1061. DOI: 10.1051/ 0004-6361:20010908.

Thomas R.J., Teske R.G. Solar soft X-rays and solar activity. II: Soft X-ray emission during solar flares. Solar Phys. 1971, vol. 16, iss. 2, pp. 431-453. DOI: 10.1007/BF00162486.

Veronig A., Temmer M., Hanslmeier A., Otruba W., Messerotti M. Temporal aspects and frequency distributions of solar soft X-ray flares. Astron. Astrophys. 2002, vol. 382, no. 3, pp. 1070-1080. DOI: 10.1051/0004-6361:20011694.

URL: http://www.ngdc.noaa.gov/stp/space-weather/solardata/solar-features/solar-flares/h-alpha/events/ (accessed September 19, 2019).

URL: https://www.ngdc.noaa.gov/stp/space-weather/solardata/solar-features/solar-flares/x-rays/goes/xrs/ (accessed September 19, 2019).

URL: https://www.ngdc.noaa.gov/stp/space-weather/solardata/solar-features/solar-flares/documentation/readme_solarfeatures_solar-flares.pdf (accessed September 19, 2019).

URL: https://sidstation.loudet.org/solar-activity-en.xhtml (accessed September 19, 2019).

URL: https://umbra.nascom.nasa.gov/SEP/ (accessed September 19, 2019).

How to cite this article

Borovik A.V., Zhdanov A.A. Low-power solar flares of optical and X-ray wavelengths for solar cycles 21-24. Solar-Terrestrial Physics. 2020. Vol. 6. Iss. 3. P. 16-22. DOI: 10.12737/stp-63202002. 